\title{
Médicaments et thermalisme à Pautalia, Thrace
}

Valentina Grigorova

\section{Summary}

The city of Pautalia (Kyustendil in Bulgaria) is located near thermal springs in the Strymon valley (Strouma), on a site occupied from the Iron Age onward by the Thracian tribe of Dentheletes. The temple of Asclepios and the walls of Pautalia, located on the hill of Hissarlaka, as well as the roman thermae in the center of modern Kyustendil are among the more important archaeological vestiges in the area. In 1990, near the village of Dragodan, district of Kyustendil, different surgical instruments in bronze were unearthed in a tumulus attributed to the roman period (IInd century A.D.). During the excavation of another tumulus in 1992, a truly exceptional discovery was made near the town of Kotcherinovo, district of Kyustendil: A variety of medicines were discovered in a small bronze case, dating from the roman period (IInd century A.D.). The complete results of the analysis of these substances and few hypotheses about their possible use are presented in this publication.

\section{Résumé}

La ville de Pautalia, située dans la vallée fertile du Strymon (Strouma), se développe à l'emplacement d'une agglomération thrace de la tribu des Dentheletes de l'âge du fer, créée à proximité d'une source thermale. Parmi les trouvailles archéologiques les plus importantes, il faut mentionner le temple d'Asclépios et les murailles de Pautalia, situées sur la colline d'Hissarlaka, ainsi que les thermes romains au centre de la ville moderne

Dr Valentina Grigorova, Université de Fribourg, Séminaire d'histoire ancienne, 16, rue PierreAeby, 1700 Fribourg. 
de Kyustendil. En 1990, dans un tumulus près du village de Dragodan, district Kyustendil, ont été trouvés des instruments chirurgicaux en bronze de l'époque romaine ( $\mathrm{II}^{\mathrm{e}} \mathrm{s}$. ap. J.-C.). Une découverte exceptionnelle, faite pendant les excavations d'un tumulus près de la ville de Kotcherinovo, district Kyustendil en 1992, a été celle d'une boîte en bronze, remplie de médicaments et datée de l'époque romaine ( $\mathrm{II}^{\mathrm{e}} \mathrm{s}$. ap. J.-C.). Dans ce texte sont présentés les résultats complets des analyses des substances trouvées à l'intérieur de la boîte, ainsi que quelques hypothèses concernant leur utilisation.

\section{Les informations archéologiques, numismatiques et épigraphiques ${ }^{1}$}

Les premières recherches archéologiques effectuées dans la ville de Kyustendil et sa région se sont déroulées entre la fin du XIX et le début du $\mathrm{XX}^{\mathrm{e}}$ s. Parmi les trouvailles les plus importantes, il faut mentionner le temple d'Asclépios et les murailles de Pautalia, situées sur la colline d'Hissarlaka $(1906,1908,1909,1911$ et 1912), ainsi que les thermes romains au centre de la ville moderne de Kyustendil (1932). Les fouilles archéologiques systématiques sur le territoire de Pautalia commencent après 1961-1962 (fig. 1).

Les fouilles archéologiques à Pautalia ainsi que les images sur ses monnaies présentent une disposition des édifices cultuels semblable à celle que Pausanias décrit pour Épidaure ${ }^{2}$. L'ensemble des monuments sur la colline d'Hissarlaka est représenté sur les monnaies de Pautalia de Septime Sévère et ses fils, Caracalla et Geta ${ }^{3}$. Sur l'image monétaire, le temple principal, celui d'Asclépios, est situé en bas, au pied de la colline, là où les fouilles archéologiques ont dégagé ses fondations. Il a été lié à un sanctuaire et à la grotte sacrée, représentée sur le flanc gauche de la colline (fig. 2). La monnaie de Caracalla montre qu'au sommet s'érigeait un temple d'Apollon, dont la présence n'est pas confirmée par les excavations ${ }^{4}$. Elles ont permis d'étudier une partie du sol du temple d'Asclépios, décoré par des mosaïques, dont un des ornements est intéressant pour notre sujet. Il s'agit d'un serpent,

1 Version écrite d'une conférence donnée dans le cadre du séminaire de médecine antique le 17 novembre 1999 à l'Institut universitaire d'histoire de la médecine et de la santé publique à Lausanne.

2 Cf. Pausanias, Description of Greece, livre II (London/Cambridge, Massachusets 1959). L'auteur décrit l'emplacement de plusieurs constructions autour du temple d'Asclépios: théâtre, temple d'Artemis et sanctuaire d'Aphrodite (II.XXVII,5), sanctuaire d'Apollon (II.XXVII, 7) etc. L'ensemble des bâtiments, à Épidaure se trouvait, comme à Pautalia, sur des collines (II. XXVII, 7).

3 Cf. L. Ruzicka, Die Münzen von Pautalia (Sofia 1933) nos 287, 713 et 908, pl. IX, nos 17 et 18.

4 Cf. L. Ruzicka, op. cit. (n. 3) n 713, pl. IX, nº 18. 


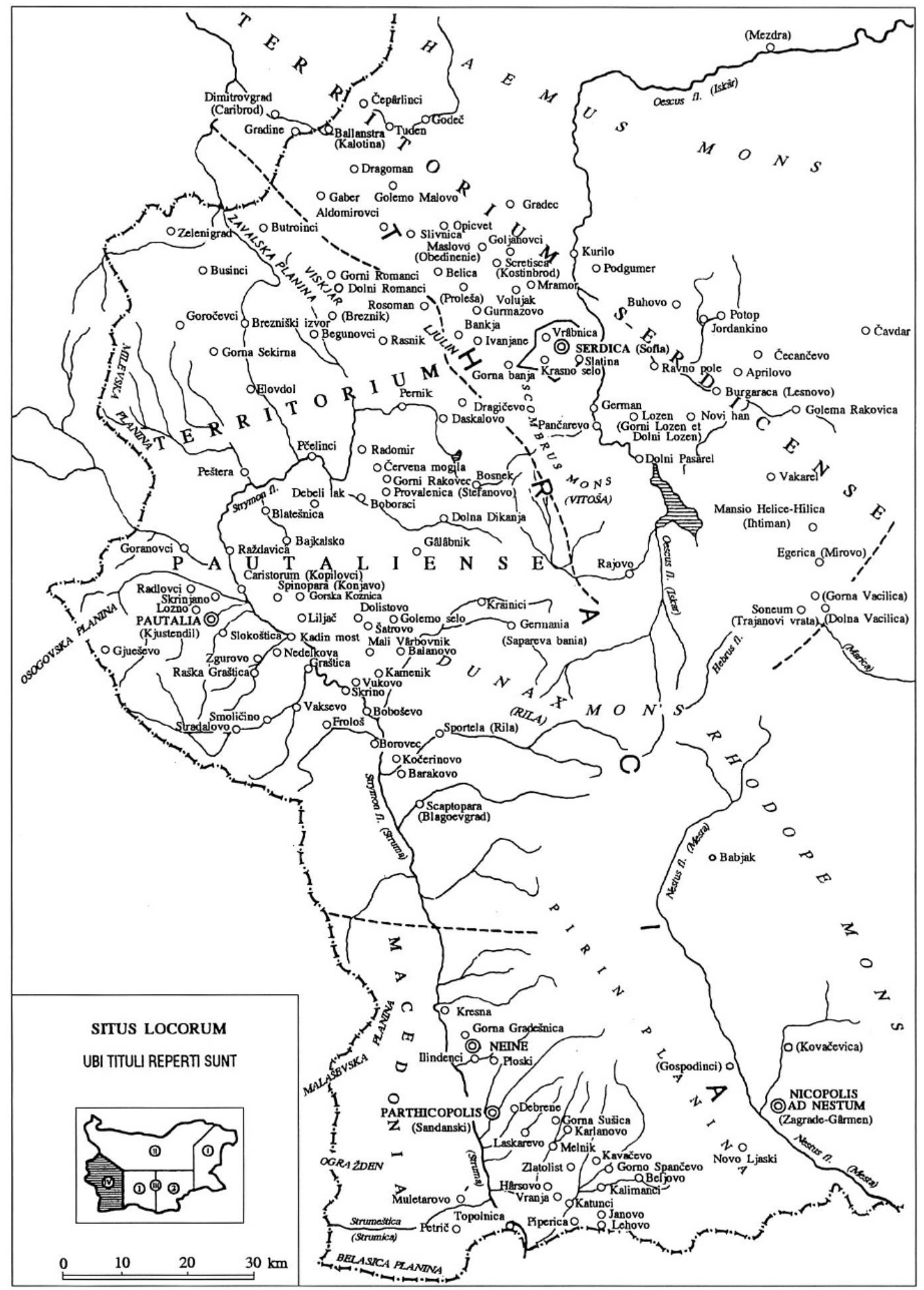

Fig. 1. Territoire de Pautalia, Thrace, d'après IGBulg V. 

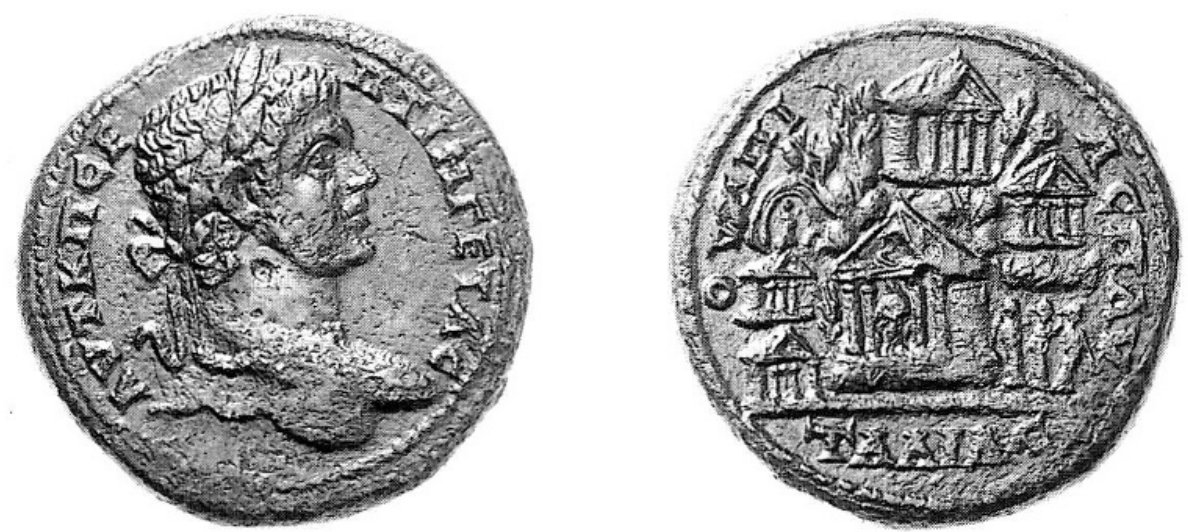

Fig. 2. Pautalia, Thrace, Geta (209-212), bronze, 30 mm, 18, 13 g, 1h. Av. AVT К П СЕПTIM

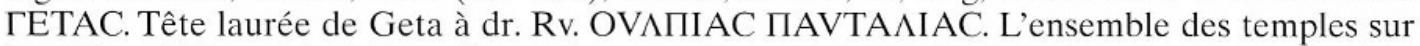
la colline d'Hissarlaka à Pautalia. En bas, au centre, le temple d'Asclépios, sa statue au milieu. A dr., les trois nymphes. A g., deux petits temples tétrastyles, au-dessus, la grotte sacrée avec une figure féminine au milieu. Au sommet, un temple tétrastyle vu de côté g., à dr., un autre temple tétrastyle. Ruzicka, Die Münzen von Pautalia (Sofia 1933) 908; Imhoof-Blumer, Nymphen und Chariten (Athen 1908) 499 var. La monnaie et la photo provient de la vente Leu 72 (Zürich, le 12 . mai $\left.1998, n^{\circ} 486\right)$.

l'attribut principal du dieu-médecin, long d'un mètre $20 \mathrm{~cm}$, représenté en blanc sur un fond bleu et dans un cadre blanc. Au même endroit a été trouvé un fragment de statue d'Asclépios en marbre blanc. Ses dimensions (hauteur et largeur du bâton $17 \times 13,5 \mathrm{~cm}$, épaisseur du serpent $5 \mathrm{~cm}$ ) nous permettent de dire que, fort probablement, il s'agit du bâton de dieu-guérisseur faisant partie de sa grande statue, érigée au milieu du temple. A proximité de l'emplacement du temple se trouvaient les thermes. Au début du $\mathrm{XX}^{\mathrm{e}}$ siècle, encore, il y avait à cet endroit des bains turcs (Shaban hamam) et à côté, le lavoir de la ville de Kyustendil, tous deux détruits pendant les travaux de captage des eaux de la source thermale. Dans les ruines dégagées pendant les travaux ont été trouvés plusieurs objets votifs. Parmi eux, une tête d'Asclépios en marbre (hauteur $5 \mathrm{~cm}$, largeur $4 \mathrm{~cm}$ ), faisant sans doute partie d'une statuette; un grand œuf en pierre $(3522 \mathrm{~kg})$ et des fragments de plusieurs tablettes votives en marbre ou en calcaire (fig. 3,4$)^{5}$. Dans le terri-

5 IGBulg IV. 2059. Tablette votive en calcaire, représentant Asclépios, Hygieia et Télesphoros, trouvée à Kyustendil. Au Musée d'histoire Kyustendil, no 13. Au dessus du relief: Kvpíos /

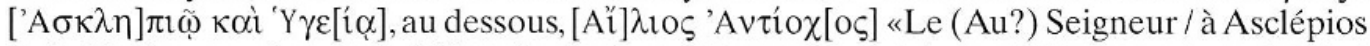
et à Hygie», au dessous "Ailios Antiochos». Normalement le mot Kyrios est au datif et s'accorde par conséquent avec le mot Asclépios, cf. les exemples qui suivent, mais ici inexplicablement il est au nominatif d'où la traduction heurtée. O. Curty, M. Piérart et T. Zawadzki ont très gentiment prêté leur concours lors de la traduction des inscriptions grecques. Qu'ils en soient chaleureusement remerciés.

IGBulg IV. 2062. Tablette votive en marbre, représentant Asclépios, trouvée dans l'Asclépieion de Pautalia. Au Musée d'histoire Kyustendil, no 288. Au dessus du relief: Kvpt⿳巛人

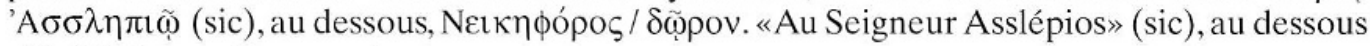
«Neikèphoros / comme don.» 


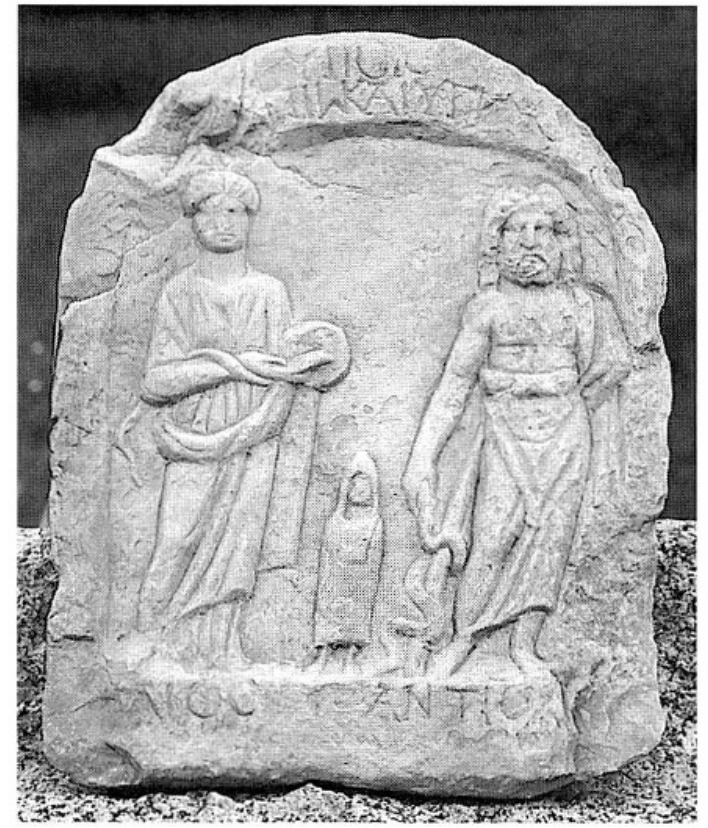

Fig. 3. Tablette votive en calcaire, représentant Asclépios, Hygieia et Télesphoros, trouvée à Kyustendil. Au Musée d'histoire Kyustendil, no 13, IGBulg IV. 2059. Au dessus

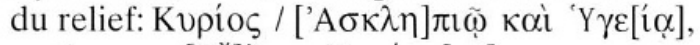

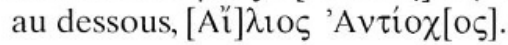

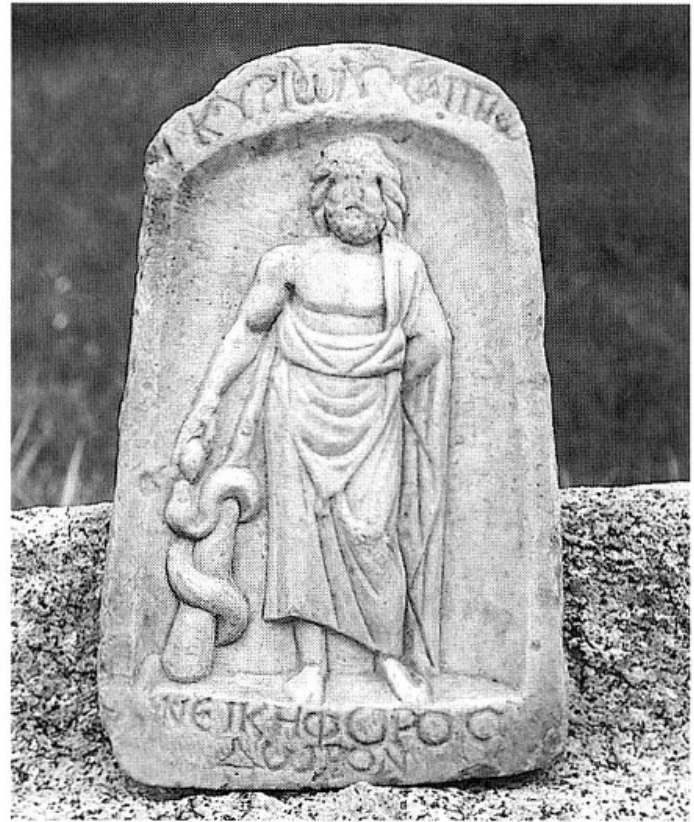

Fig. 4. Tablette votive en marbre, représentant Asclépios, trouvée dans l'Asclépieion de Pautalia. Au Musée d'histoire Kyustendil, $n^{\circ} 288$, IGBulg IV. 2062. Au dessus du relief: Kvpí $\omega$ / 'A $\sigma \sigma \lambda \eta \pi \iota(\tilde{)}$ (sic), au dessous,

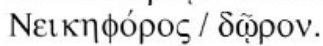

toire de Pautalia, près du village d'Eremia, fut découverte une statuette d'Asclépios en bronze d'une fabrication exquise ${ }^{6}$. Il est très intéressant de remarquer la continuité du culte d'Asclépios confirmée par la trouvaille de dizaines de couteaux en fer dans la source thermale. Les derniers étaient jetés dans l'eau après l'égorgement de l'animal de sacrifice, communément, un coq. Cette coutume a été respectée à Kyustendil jusqu'aux années $20^{7}$.

Les fouilles d'urgences en 1962-1964 ont permis de découvrir et étudier environ $1000 \mathrm{~m}^{2}$ de l'Asclépieion de Pautalia (fig. 5). Les constructions qui continuent, malheureusement, sous les bâtiments modernes occupent fort probablement ca. $3000 \mathrm{~m}^{2} .^{8}$ Les salles découvertes par la fouille ont été identifiées comme faisant partie de thermes, notamment un apodyterium,

6 Cf.J. Meshekov, L.Staikova,Sculpture antique en bronze II-IIIs. (en bulg. Antitchna bronzova plastika II-III v.) (Sofia 1997) 21. Au Musée d'histoire Kyustendil, no 900 . Hauteur 6,5 cm, II ${ }^{\mathrm{e}}$ s. ap. J.-C.

7 Cf. J. Ivanov, «Le Hissarlak de Kyustendil et ses antiquités» (en bulg. «Kyustendilskiyat Hisarlak i negovite starini»), Bulletin de la société archéologique bulgare (en bulg. Izvestiya na balgarskoto archeologichesko druzestvo) 7 (1919-1920) 73.

8 Cf. L. Rousseva-Slokoska, «Un bâtiment public romain de Pautalia» (en bulg. «Rimska obschtestvena sgrada v Pautalia»), Archeologiya 6,1 (1964) 33ff. 


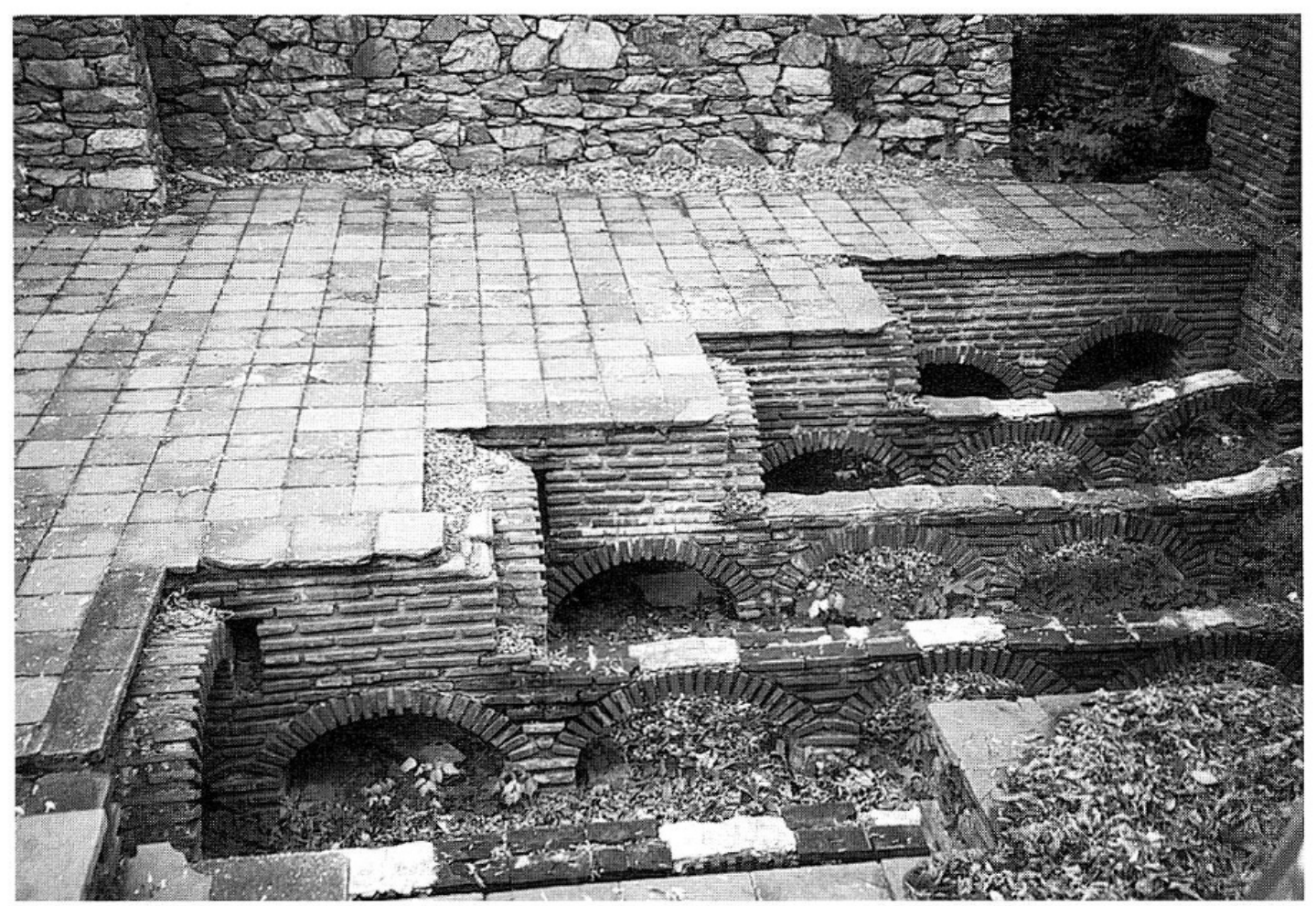

Fig. 5. Les thermes de Pautalia (II $-\mathrm{III}^{\mathrm{e}}$ s. ap. J.-C.).

un frigidarium, un tepidarium et un caldarium. Certaines salles disposent d'hypocaustes et de piscines. La construction est de type opus mixtum, dont les lits de petits blocs de pierre alternent d'une manière régulière avec quatre épaisseurs de briques. Le sol est revêtu de marbre, les murs, ornés de corniches profilées et de pilastres. Les grandes salles sont reliées par des passages avec seuil en granit, portant des traces de portes à deux battants. La datation du bâtiment est basée sur une trouvaille de 61 deniers romains d'Antonin le Pieux jusqu'à Gordien III ainsi que sur de nombreux fragments de céramiques et de tablettes votives.

Pautalia a son atelier monétaire qui a fonctionné presque sans interruption du temps de l'empereur Antonin le Pieux (138-161) jusqu'au règne de l'empereur Caracalla (198-217). Sur les monnaies pautaliennes, on trouve de nombreuses représentations qui reflètent le thermalisme et le culte de divinités de la santé. Sur leur revers, les monnaies portent, très fréquemment, les images d'Asclépios, Hygieia et Télesphoros; des nymphes et des serpents, ainsi que celle du temple d'Asclépios sur la colline d'Hissarlaka, liées aux sources thermales à Pautalia. Dans son Corpus du monnayage de Pautalia, édité en 1933, L. Ruzicka publie plusieurs de ces types. Des nouveaux se sont rajoutés à la suite de mes recherches pendant les années 1990. 
Une inscription d'Épidaure montre que le culte de divinités de la santé de Pautalia était connu hors du territoire de la ville et de la Thrace9 ${ }^{9}$ Quatre autres inscriptions étaient dédiées à Asclépios et Hygieia par de fervents adeptes de leur culte, citoyens de Pautalia, dans le sanctuaire d'Asclépios près du village de Glava Panega, District Lovetch ${ }^{10}$. Une très intéressante inscription, trouvée près du village de Konyavo (Spinopara), district Kyustendil, nous donne la liste des néocores dans le temple d'Asclépios Soter ${ }^{11}$. Dans le village de Spinopara se trouvaient les terrains, mentionnées dans cette inscription, appartenant au temple. A ma connaissance, c'est le seul document épigraphique de la Thrace qui atteste l'existence de propriétés religieuses. En ce qui concerne les néocores d'après le texte on peut dire avec certitude qu'ils étaient organisés dans un collège, présidé par un $\pi \alpha \tau \eta ̀ \rho$. Hélas, l'absence d'autres informations a ce sujet ne permet pas de savoir si les néocores participaient aux pratiques médicales comme, par exemple, à Épidaure.

9 IG IV. 477. Épidaure, Grèce. 'A $\sigma \kappa \lambda \eta \pi \imath \tilde{\omega} \Sigma \omega \tau \eta \tilde{\rho l, ~ ' Y \gamma l \varepsilon i ́ \alpha l, ~ Т \varepsilon \lambda \varepsilon \sigma \phi o ́ p \omega ~ П \alpha v \tau \alpha \lambda ı \omega \tau \alpha ı \varsigma ~}$

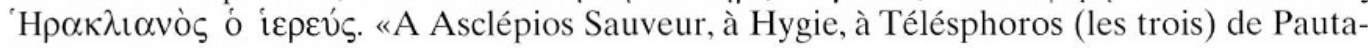
lia, le prêtre Hèraklianos.»

10 IGBulg II. 511. Glava Panega près de la ville de Teteven, District Lovetch. 'A $\sigma \kappa \lambda \eta \pi \iota \widetilde{\omega}$

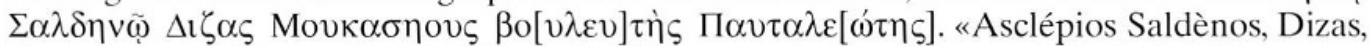
fils de Moukasèès, bouleute de Pautalia.» Le terme de bouleute est difficile à définir selon les institutions modernes. Il s'agit èn quelque sorte d'un membre du conseil de la cité.

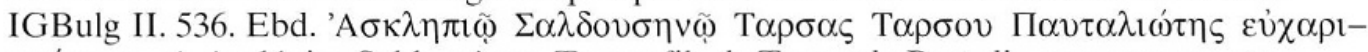

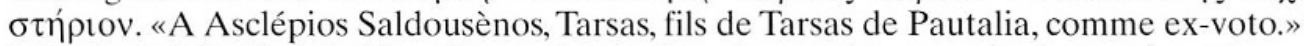

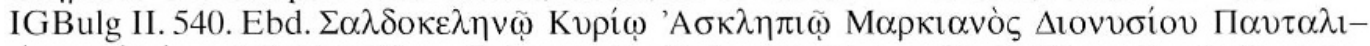

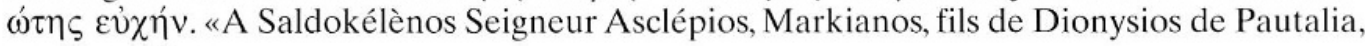
comme prière.»

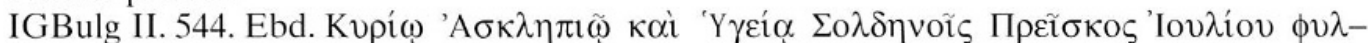

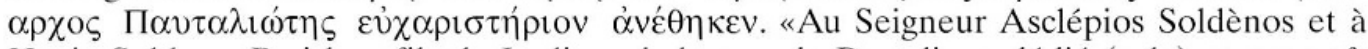
Hygie Soldene, Preiskos, fils de Ioulios, phylarque de Pautalia, a dédié (cela) comme offrande.» Le terme de phylarque indique à l'origine le chef d'une division de la population. Il s'agit d'un des magistrats de la cité.

11 IGBulg IV. 2192. Plaque en marbre, trouvée près du village de Konyavo, district Kyustendil.

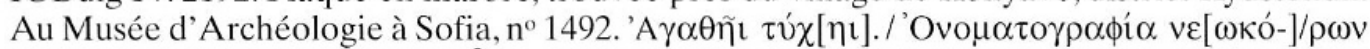

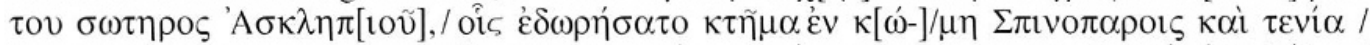

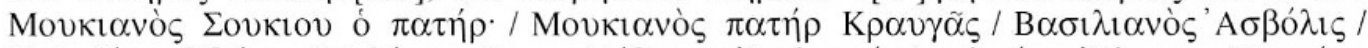

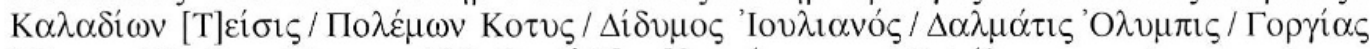

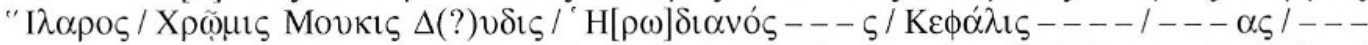
$\imath \pi \mathrm{\varsigma} /$ - - - «A la bonne Fortune / nomenclature de néocores du Seigneur Asclépios, / auxquels Moukianos, fils de Soukios le chef (des néocores) / a donné un domaine dans le village de Spinopara et des terres (?) / Moukianos le chef / Kraugas / Basilianos Asbolis / Kaladion Teisis / Polémôn Kotys / Didymos Ioulianos / Dalmatis Olympis / Gorgias Ilaros / Chrômis Moukis D(?)ydis / Hérodianôs -s / Képhalis / -as / -ipis /----.» Le terme de néocore signifie une sorte de prêtre. 


\section{Les découvertes récentes}

Des instruments chirurgicaux en bronze de l'époque romaine ( $\mathrm{II}^{\mathrm{e}} \mathrm{s}$. ap. J.-C.) ont été trouvés en 1990 dans un tumulus près du village de Dragodan, district Kyustendil. Il s'agit d'un scalpel, de deux curettes et d'une plaquette en pierre pour la préparation de divers médicaments (fig. 6,8 ) $^{12}$. Une autre découverte, d'une importance majeure, a été celle d'une boîte en bronze, remplie de médicaments et datée de l'époque romaine ( $\mathrm{II}^{\mathrm{e}} \mathrm{s}$. ap. J.-C.) (fig. 7). Elle a été trouvée pendant les excavations d'un tumulus près de la ville de Kotcherinovo, district Kyustendil en 1992, jointe à une couronne funéraire en or, deux bagues en or serties des gemmes, 13 monnaies romaines en argent, de la vaisselle en bronze et de divers objets en fer, en céramique et en verre ${ }^{13}$. Il est intéressant de signaler que le bûcher funéraire était entouré par deux grands cercles $(4 \times 5 \mathrm{~m})$, l'un formé par de la vaisselle en céramique, l'autre, par des objets en bronze et en fer. En raison de la rareté de pareilles découvertes, il a semblé important d'effectuer une analyse des substances trouvées

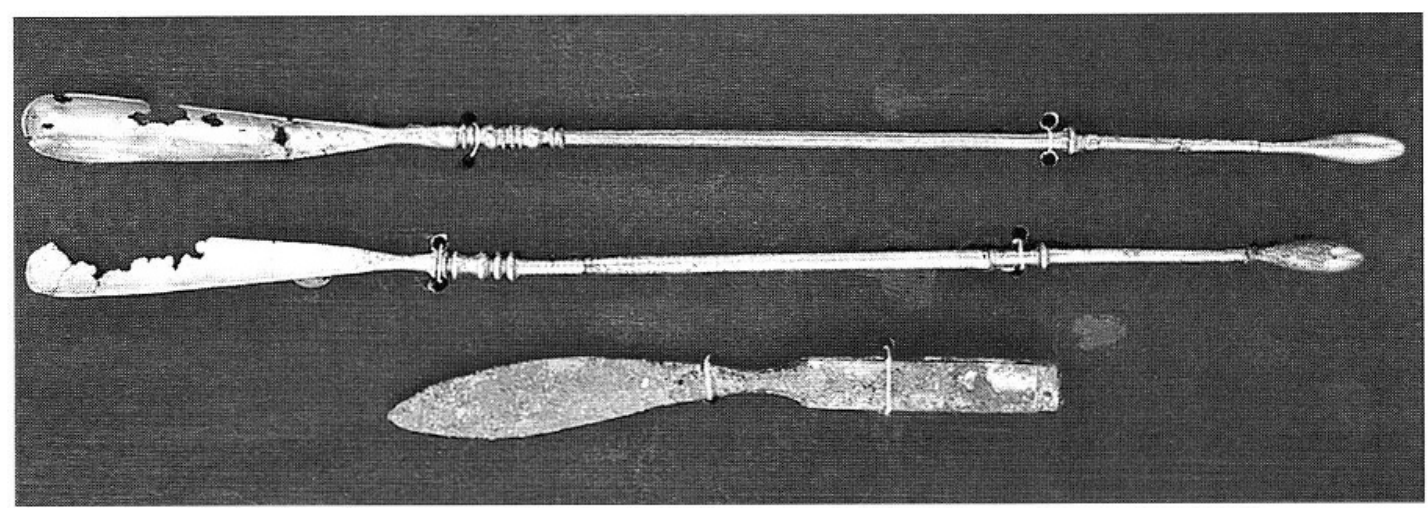

Fig. 6. Instruments chirurgicaux en bronze (II ${ }^{\mathrm{e}}$ s. ap. J.-C.), Musée d'histoire à Kyustendil, Bulgarie.

12 Les instruments ont été trouvés ensemble avec une couronne funéraire en or, une bague en or, 39 deniers romains, de la vaisselle en argent et des objets en bronze. Ils sont inventoriés au Musée d'histoire Kyustendil: le scalpel sous n² 246 , les curettes, nos 247 et 248, la plaquette, no 315. Cf. N. Theodossiev/M. Manov, «Le tumulus près du village de Dragodan, District Kyustendil» (en bulg. «Mogilno pogrebenie pri s. Dragodan, Kyustendilsko»), Archeologiya 35,1 (1993) 31-42.

13 Les fouilles archéologiques ont été dirigées par les Drs. A. Boshkova et P. Delev. Cf. A. Boshkova, «Recherches archéologiques dans la région de Kotcherinovo» (en bulg. "Archeologicheski proutchvaniya v rayona na Kotcherinovo»), Bulletin du Musée d'histoire Kyustendil 4 (1992) 245-252. La boîte n'est pas encore restaurée et se trouve au Musée d'histoire Kyustendil. 

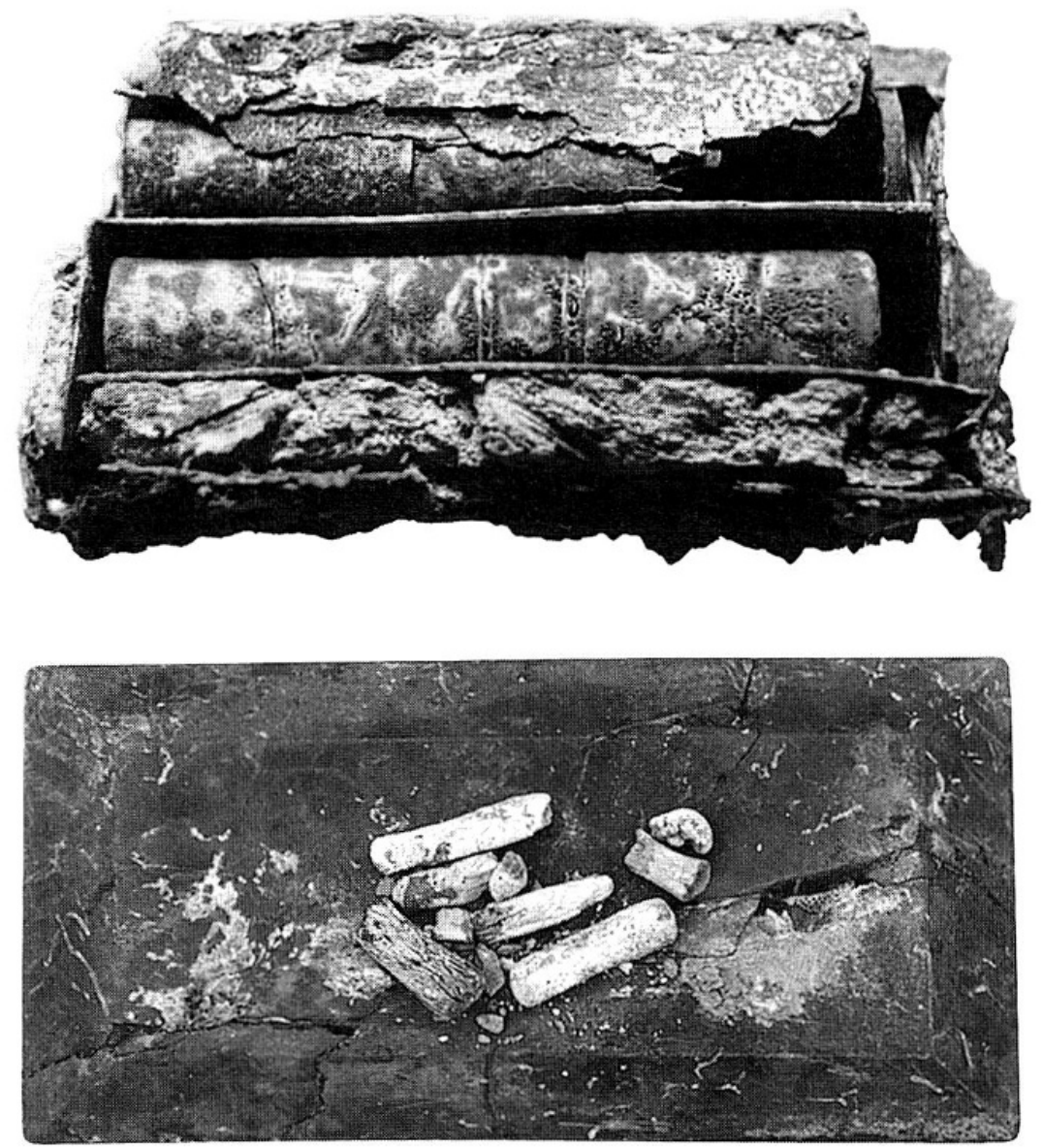

Fig. 7. Boîte en bronze, remplie de médicaments (II ${ }^{\mathrm{e}}$ s. ap. J.-C.), Musée d'histoire à Kyustendil, Bulgarie.

Fig. 8. Comprimés romains sur une plaquette en pierre pour préparation des divers médicaments.

à l'intérieur de la boîte en bronze ${ }^{14}$. La seule trouvaille analysée du territoire de la Bulgarie contenant de médicaments et de produits cosmétiques antiques provient des excavations d'une tombe romaine près de la ville de Bansko, district Blagoevgrad en 1970. Elle contient une boîte en bronze avec des médicaments, des instruments chirurgicaux et un récipient en bronze, en forme de bottine, remplie d'un mélange aromatique ${ }^{15}$.

14 Nos remerciements vont au Laboratoire de toxicologie analytique de l'Institut universitaire de médecine légale à Lausanne et spécialement au Dr Christian Giroud ainsi qu'à la Société SCITEC SA à Lausanne et à son directeur M. Ivan Slacanin.

15 Cf. Z. Dimitrova, «The heirs of Orpheus. Bulgarian medicine and pharmacy over the centuries», dans: The Pharmacy. Windows on History (Basel 1996) 91-100 et M. Apostolov/Sv. Atanassova, «Analyse de contenu des médicaments trouvés dans une tombe romaine près de Bansko» (en russe «Izutchenie sostava lekarstv, naydenich v rimskoy grobnice okolo goroda Bansko»), dans: Asklepyi, vol. III (Sofia 1974) 55-65. 


\section{Les analyses}

Les substances à analyser étaient indiquées par des lettres A (1 morceau de $108 \mathrm{mg}$, couleur brun-roux), B (trois petits morceaux verdâtres pesant $273 \mathrm{mg}$ ) et C (deux morceaux brunâtres à l'extérieur, vert à l'intérieur et de la poudre d'un poids total de $209 \mathrm{mg}$ ) (fig. 8). Une chromatographie en phase gazeuse a été pratiqué au Laboratoire de toxicologie analytique pour séparer les composants de ces trois échantillons. L'application de la spectrométrie de masse a rendu possible leur identification. Elle nous a donné des informations concernant la nature, la composition et la structure des espèces présentées dans les substances analysées. Les recherches de molécules organiques se sont avérées négatives, aucune substance n'étant détectée. La radiographie des trois échantillons montre une structure radioopaque qui suggère de produits constitués de matière inorganique, de minéraux. Les composants inorganiques ont été analysés dans le laboratoire de la société SCITEC en utilisant l'excitation par torche à plasma inductif. Cette procédure où les substances subissent des températures proches de $8000^{\circ} \mathrm{K}$ permet d'identifier les éléments chimiques par la couleur du plasma dépendant du gaz qui lui donne naissance. Les résultats complets des analyses de cations sont présentés dans un tableau (fig. 9). Plus bas, je mentionne uniquement les éléments principaux de chacune de nos trois substances. Dans la composition de l'échantillon A prédomine le plomb $(151,8 \mathrm{~g} / \mathrm{kg})$; on observe également du zinc $(69,7 \mathrm{~g} / \mathrm{kg})$, du calcium $(43,7 \mathrm{~g} / \mathrm{kg})$, du fer $(38,6 \mathrm{~g} / \mathrm{kg})$ et du cuivre $(31,3 \mathrm{~g} / \mathrm{kg})$. La substance $\mathrm{B}$ contient une quantité très importante de plomb $(612,0 \mathrm{~g} / \mathrm{kg})$ ainsi que relativement peu de zinc $(1 \mathrm{~g} / \mathrm{kg})$ et de cuivre $(0,9 \mathrm{~g} / \mathrm{kg})$. L'échantillon $\mathrm{C}$ présente une structure mélangée avec prédominance de plomb $(257,5 \mathrm{~g} / \mathrm{kg})$, d'arsenic $(94,8 \mathrm{~g} / \mathrm{kg})$, de cuivre $(85,4 \mathrm{~g} / \mathrm{kg})$ et de zinc $(39,6 \mathrm{~g} / \mathrm{kg})$, contenant également du fer $(7,1 \mathrm{~g} / \mathrm{kg})$, du baryum $(5,5 \mathrm{~g} / \mathrm{kg})$ et de l'aluminium $(4,5 \mathrm{~g} / \mathrm{kg})$. A cause de la très faible quantité de substances à analyser, le mercure n'est pas détectable.

Les résultats des analyses de médicaments trouvés près de Kotcherinovo sont semblables à celles des médicaments trouvés dans la tombe romaine près de Bansko, également dans le territoire de Pautalia ${ }^{16}$. Cette constatation est extrêmement réjouissante, car elle montre que ces médicaments étaient, fort probablement, produits dans un seul et même centre médical, en l'occurence celui à Pautalia, particulièrement célèbre pendant l'époque romaine. Les deux trouvailles contiennent avant tout du plomb, du zinc et du cuivre. Dans leur composition il y a également du calcium et du fer. La seule grande

16 Cf. note 15, M. Apostolov/Sv. Atanassova, op. cit. (n. 15) 62-64. 
différence est la présence d'une quantité importante d'arsenic dans l'échantillon $\mathrm{C}$ des médicaments trouvés près de Kotcherinovo. Une telle composition chimique correspond bien aux applications de ces éléments dans les procédures médicales pendant l'Antiquité. Le livre De Medicina de Celse, nous a servi de guide dans la pharmacie. On peut y lire que le plomb, l'oxyde de plomb et les minerais de cuivre étaient utilisés pour arrêter les hémorra-

Fig. 9. Teneur en métaux dans les comprimés romains (* $\mathrm{ppm}=\mathrm{mg} / \mathrm{kg}$ )

\begin{tabular}{|c|c|c|c|}
\hline Elément & $\begin{array}{l}\text { Stu } 4542 \text { A } \\
\text { ppm* }\end{array}$ & $\begin{array}{l}\text { Stu } 4543 \text { B } \\
\text { ppm }\end{array}$ & $\begin{array}{l}\text { Stu } 4544 \mathrm{C} \\
\text { ppm }\end{array}$ \\
\hline As & $<0.5$ & $<0.5$ & 94849 \\
\hline $\mathrm{Ag}$ & 35.6 & 8.6 & $<0.1$ \\
\hline $\mathrm{Al}$ & 4989 & 18.2 & 4476 \\
\hline B & $<0.5$ & $<0.5$ & $<0.5$ \\
\hline $\mathrm{Ba}$ & 75.0 & 11.0 & 5471 \\
\hline $\mathrm{Bi}$ & $<0.5$ & $<0.5$ & $<0.5$ \\
\hline $\mathrm{Ca}$ & 43710 & 108 & 3037 \\
\hline $\mathrm{Cd}$ & $<0.1$ & $<0.1$ & 664 \\
\hline Co & $<0.1$ & $<0.1$ & $<0.1$ \\
\hline $\mathrm{Cr}$ & $<0.1$ & $<0.1$ & $<0.1$ \\
\hline $\mathrm{Cu}$ & 31343 & 877 & 85435 \\
\hline $\mathrm{Fe}$ & 38600 & 21.9 & 7087 \\
\hline $\mathrm{Ga}$ & $<0.1$ & $<0.1$ & $<0.5$ \\
\hline In & $<0.1$ & $<0.1$ & $<0.5$ \\
\hline $\mathrm{K}$ & 456 & 23.5 & 719 \\
\hline $\mathrm{Li}$ & $<0.1$ & $<0.1$ & $<0.1$ \\
\hline $\mathrm{Mg}$ & 13200 & 16.3 & 1226 \\
\hline $\mathrm{Mn}$ & 375 & 6.2 & 115 \\
\hline $\mathrm{Na}$ & 942 & 228 & 1554 \\
\hline $\mathrm{Ni}$ & 29.2 & $<0.1$ & 17.8 \\
\hline $\mathrm{Pb}$ & 151800 & 612058 & 257549 \\
\hline $\mathrm{Sb}$ & $<0.5$ & $<0.5$ & $<0.5$ \\
\hline $\mathrm{Sr}$ & 520 & 8.1 & 101 \\
\hline $\mathrm{Te}$ & $<0.1$ & $<0.1$ & $<0.1$ \\
\hline $\mathrm{Zn}$ & 69700 & 978 & 39609 \\
\hline
\end{tabular}


gies ainsi que comme laxatif; les minerais de zinc, quant à eux, étaient utilisés dans la guérison des maladies oculaires. Le minerai de cuivre permettait également de nettoyer et cicatriser les plaies. Identique était l'utilisation des sulfates et des oxydes de fer ${ }^{17}$. Le calcium sous forme de chaux était appliqué comme moyen pour brûler les tissus malades, sous forme de gypse pour baisser la fièvre et comme antisudora ${ }^{18}$. Les Thraces utilisaient aussi des remèdes contenant de l'oxyde de zinc. Un composé de ce groupe se trouve sous le nom calaminthae parmi les médicaments mentionnés par le médecin romain Dioscoride ${ }^{19}$.

L'analyse d'une quantité plus important de substances permettrait de détecter la présence ou l'absence d'encore quelques éléments chimiques utilisés dans les pratiques médicales anciennes. D'autre part, une analyse détaillée d'anions permettrait de trouver la forme exacte sous laquelle se trouvent les différents composants de nos substances. Ces futures recherches liées à l'ensemble des informations archéologiques, numismatiques et épigraphiques nous permettront de tenter une reconstitution théorique des activités du centre thermal et médical à Pautalia pendant l'époque romaine.

17 Cf. Celsius, De Medicina (Pisa 1975) V, 1, 5, 6, 9; VI, 6, 5.

18 Op. cit., V, 6; II, 33.

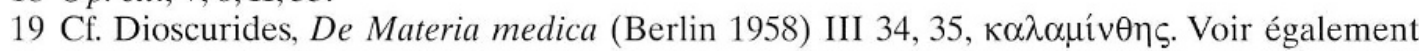
A. Schmidt, Drogen und Drogenhandel im Altertum (New York 1979) 10-11 et note 15, Z. Dimitrova, op. cit. (n. 15) 94. 